\title{
Intraoperative radiation therapy for breast cancer patients: current perspectives
}

\author{
This article was published in the following Dove Press journal: \\ Breast Cancer - Targets and Therapy \\ I8 April 2017 \\ Number of times this article has been viewed
}

\author{
Sunil W Dutta' \\ Shayna L Showalter ${ }^{2}$ \\ Timothy N Showalter' \\ Bruce Libby' \\ Daniel M Trifiletti' \\ 'Department of Radiation Oncology, \\ ${ }^{2}$ Division of Surgical Oncology, \\ Department of Surgery, University \\ of Virginia School of Medicine, \\ Charlottesville, VA, USA
}

\begin{abstract}
Accelerated partial breast irradiation (APBI) provides an attractive alternative to whole breast irradiation (WBI) through normal tissue radiation exposure and reduced treatment duration. Intraoperative radiation therapy (IORT) is a form of APBI with the shortest time interval, as it delivers the entirety of a planned radiation course at the time of breast surgery. However, faster is not always better, and IORT has been met with healthy skepticism. Patients treated with IORT have an increased compliance and overall satisfaction when compared to patients treated with WBI. However, early randomized trial results demonstrated an increased rate of recurrence after IORT, slowing its widespread adoption. Despite these controversies, IORT utilization is increasing nationally and several novel developments are aimed at continuing to minimize the risk of recurrence and treatment-related toxicity while maximizing the patient experience.

Keywords: IORT, lumpectomy, breast conservation, electron, photon, evidence
\end{abstract}

\section{Introduction}

For patients with early-stage breast cancer, breast-conserving therapy (BCT) is commonly the preferable surgical treatment option because of the demonstrated equivalence in survival when compared to total mastectomy. ${ }^{1}$ BCT mostly consists of lumpectomy followed by adjuvant radiation therapy. Radiation therapy in the form of whole breast irradiation (WBI) is typically given daily over 5 weeks and reduces the likelihood of recurrence in the ipsilateral breast. ${ }^{2}$ Despite the demonstrated benefits of adjuvant radiotherapy, up to a quarter of women who undergo lumpectomy do not receive postoperative breast irradiation. ${ }^{3}$ Increased age and long-distance travel to the radiotherapy center have been identified as determinants of omission of radiation therapy, suggesting that optimizing the patient experience can serve to improve radiotherapy utilization and as a result improve disease control. ${ }^{4,5}$ Accelerated partial breast irradiation (APBI), which involves delivering a higher dose of radiation to a smaller volume of breast tissue over a shorter period of time, has been proposed as an alternative to WBI. The American Society for Radiation Oncology (ASTRO) and other professional societies have issued consensus criteria for APBI suitability, which usually include small $(\leq 3 \mathrm{~cm})$, node-negative breast cancers with negative surgical margins, age of at least 50 years old, and patients with non-invasive disease. ${ }^{6-8}$

Intraoperative radiation therapy (IORT) is a type of APBI delivered in a single fraction after lumpectomy and before surgical wound closure. IORT offers many logistical advantages to WBI and APBI, including patient convenience, decreased irradiation of healthy organs, and patient compliance among others. Despite the theoretical and demonstrated advantages and disadvantages of IORT, it has been met with healthy 
skepticism. This review aimed to describe the existing evidence supporting the proposed advantages of IORT, as well as to describe its potential future directions to expand its acceptance and utilization.

\section{Evidence supporting IORT The ELIOT trial}

Two large, prospective randomized trials comparing IORT to WBI have been performed, each demonstrating technical differences in their approach to IORT. The ELIOT trial used highly energized electrons to deliver radiotherapy to the lumpectomy cavity during surgery. ${ }^{9}$ The ELIOT trial randomized 1305 patients, who were $\geq 48$ years old and had a tumor size $\leq 2.5 \mathrm{~cm}$ to either a single dose of 21 Gy intraoperatively or 50 Gy of WBI with a 10 Gy boost over 6 weeks. The electron technique involved a dedicated linear accelerator used to create a collimated electron beam with 6-9 MeV energies, with $21 \mathrm{~Gy}$, prescribed to the $90 \%$ isodose, given to the tumor bed after lumpectomy. The 5-year ipsilateral breast tumor recurrence (IBTR) was significantly higher in the intraoperative electron radiation therapy (IOERT) arm compared to the WBI arm $(4.4 \%$ vs $0.4 \%, p=0.0001)$. In addition, outside of the prespecified equivalence margin, 14 patients developed new ipsilateral breast carcinomas in the IOERT arm compared to 0 in the WBI arm. However, for women in the study defined as ELIOT low risk (tumor size $\leq 2 \mathrm{~cm}, \leq 3$ positive nodes, grade 1 or 2 , estrogen receptor positive, and not triple negative disease, $n=407$ ), IBR was shown to be $1.7 \%$ at 5 years. This subgroup comprises the majority of patients now considered appropriate for IORT.

Overall toxicity profile was lower in the IOERT group. Skin erythema, dryness, hyperpigmentation, and pruritus were all significantly lower compared to WBI. A subgroup of patients volunteered to undergo follow-up spiral computed tomography (CT), and the pulmonary fibrosis rate was higher in the WBI group. Fat necrosis, however, occurred at a higher rate in the IOERT group $(17 \%$ vs $7 \%, p=0.04)$.

\section{The TARGIT trial}

The Targeted Intraoperative Radiation Therapy (TARGIT-A) trial randomized 3451 patients, who were $\geq 45$ years old with a $\leq 3.5 \mathrm{~cm}$ unifocal invasive ductal carcinoma to either $20 \mathrm{~Gy}$ in the tumor bed or 50 Gy over 3-5 weeks with or without a lumpectomy bed boost depending on institutional preference. ${ }^{10}$ The TARGIT technique to deliver IORT involves placement of a $1.5-5.0 \mathrm{~cm}$ spherical applicator in the tumor bed following lumpectomy. An intrabeam device (Carl Zeiss Meditec, Jena, Germany) is used to deliver $50 \mathrm{kV}$ energy X-rays at the volume surrounding a spherical applicator implanted into the lumpectomy cavity. The surface of the tumor bed receives $20 \mathrm{~Gy}$ and attenuates to 5-7 Gy at $1 \mathrm{~cm}$ depth. Initial results publication found that the 5-year overall recurrence risk was higher in the TARGIT-A patients compared to external beam radiation therapy (EBRT) (hazard ratio [HR] 1.44, $p=0.053$ ). The ipsilateral breast recurrence risk at 5 years was also higher in the IORT $\operatorname{arm}(3.3 \%$ vs $1.3 \%, p=0.042)$. Due to risk factors during surgery or those seen on final pathology, $21 \%$ of patients who received IORT in the prepathology arm (IORT delivered before final pathology was available) also received 50 Gy of EBRT. The protocol recommended, but did not require, EBRT for close margins $(<1 \mathrm{~mm})$, extensive in situ component, or unexpected invasive lobular carcinoma. The prepathology group met the trial's noninferiority margin of $2.5 \%$, while the postpathology group (IORT not delivered at time of lumpectomy but within 30 days) did not. TARGIT-A trial authors discussed that the microenvironment of a fresh tumor bed with well-vascularized tissues may have been an advantage for the prepathology cohort and may explain the higher local recurrence rate in the otherwise well-selected postpathology group.

In the TARGIT trial, grade 3-4 skin toxicities were less in the IORT group than in the WBI group $(0.2 \%$ vs $0.8 \%$, $p=0.029)$. Also of note, fewer non-breast cancer-related deaths were reported in the IORT group $(1.4 \%$ vs $3.5 \%, p=0.0086)$, which the authors attributed to cardiac events and other cancers due to WBI. In an analysis of long-term toxicities, when comparing the IORT group to the WBI group, similar rates of fibrosis, breast edema, retraction, lymphedema, and pain were reported. ${ }^{11}$ No telangiectasia were seen after IORT alone; they were seen at a similar rate $(17 \%)$ for patients who received IORT + WBI or WBI. Furthermore, significantly less breast and arm symptoms were reported in patients who received IORT alone.

\section{CT-HDR-IORT}

The University of Virginia Health System developed a novel method of IORT termed precision breast IORT (PB-IORT). PB-IORT combines high dose rate brachytherapy and CT imaging to deliver a single dose of customized radiation at the time of lumpectomy and has been shown to be safe and feasible. ${ }^{12}$ An ongoing Phase II trial has been designed to assess the efficacy in comparison to conventional TARGIT style IORT.

PB-IORT occurs in an integrated brachytherapy suite that is equipped with full anesthesia capability and CT-on-rails technology. During the procedure, the patient first undergoes a lumpectomy (prepathology cohort) or reopening of the lumpectomy cavity (postpathology cohort). Next, a multicath- 
eter brachytherapy balloon is placed into the lumpectomy bed. CT images are taken and reviewed by the surgeon, radiation oncologist, and physicist. If needed, surgical adjustments can then be made in order to increase the conformity between the balloon and the breast tissue. Next, a computerized treatment plan is created, and the dose is able to be sculpted away from the skin and chest wall if necessary. A dose of 12.5 Gy is delivered in a single fraction prescribed $1 \mathrm{~cm}$ from the balloon surface using an iridium-192 high dose-rate (HDR) brachytherapy source (Figure 1). The balloon is then removed and the skin incision is closed. Eligibility criteria for the Phase I study included women aged $\geq 45$ years with tumors $<3 \mathrm{~cm}$ (ductal carcinoma in-situ or invasive cancer) and no pathologic evidence of nodal metastases. If patients in the prepathology cohort were found to have positive margins, then re-excision lumpectomy followed by WBI to 45 Gy in 25 daily fractions is recommended. In the Phase I study, no grade 3+ toxicities were reported, and majority (93\%) of the 28 patients had good-to-excellent cosmetic outcomes at last follow-up. This HDR brachytherapy technique was compared to the TARGIT trials $50 \mathrm{kV}$ X-ray technique and was found to have superior dosimetry on all parameters related to target volume dosing. ${ }^{13}$ Although within predefined tolerance, mean heart and rib dose were nominally increased compared to the superficial photon technique. ${ }^{13}$ Although there have been no patients with disease recurrence on this trial, follow-up duration remains limited.

\section{Patient experience}

IORT improves patient convenience and subsequently compliance when compared to WBI. Analysis of 1903 breast cancer patients who underwent BCS found a fivefold increased risk of local recurrence in patients noncompliant with guideline recommendations for radiotherapy. ${ }^{14}$

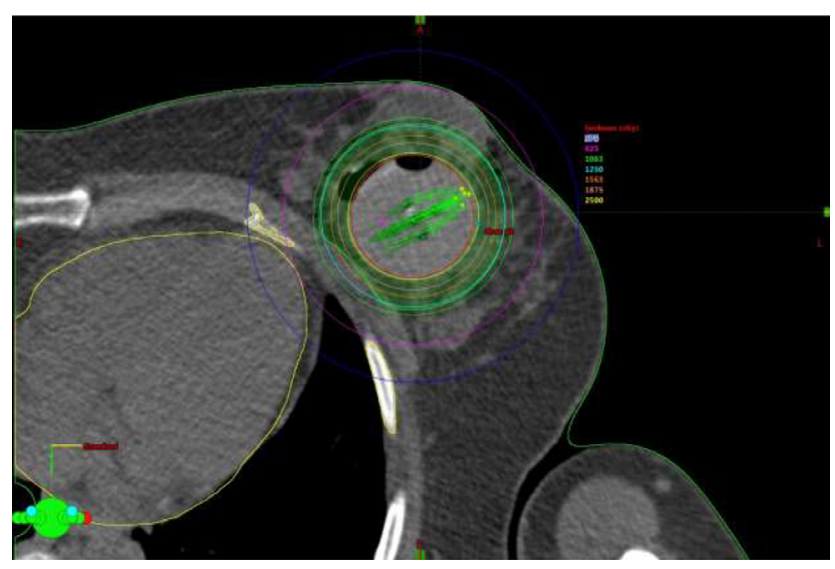

Figure I A representative axial CT image of a patient treated on the PB-IORT protocol.

Abbreviation: PB-IORT, precision breast intraoperative radiation therapy.
It is reasonable to suspect that IORT would result in less toxicity compared to WBI, owing to the fact that in WBI the entirety of the breast is targeted, while in APBI only the highest risk volume is. Five-year results from the TARGITA trial found IORT to be equivalent to WBI with regard to patient-reported cosmetic outcomes and IORT patients had superior breast-related quality-of-life (QOL) outcomes. ${ }^{15,16}$ These breast symptoms include worse pain, swelling, oversensitivity, and skin problems. Similarly, compared with WBI, the ELIOT trial demonstrated less skin toxicities, which include erythema, dryness, hyperpigmentation, or itching in the IOERT cohort. ${ }^{9}$ Taken as a whole, these results provide prospective, randomized data that confirm the suspicion that volume of breast irradiated (ie, the whole breast compared to a portion of the breast) is closely tied to subsequent toxicity and long-term cosmesis. Moreover, these results confirm that this improvement results in a difference for patients on a practical level (QOL measures).

Grade 3 and Grade 4 radiation-related skin complications were fewer in the TARGIT trial patients treated with IORT likely due to the smaller treatment volume. However, due to high single-fraction dose in the IOERT arm, fat necrosis and fibrosis rates have been reported at $4.2 \%$ and $1.8 \%$, respectively. ${ }^{17}$ Fat necrosis, which can interfere with surveillance imaging, can lead to palpable breast mass, pain, and skin changes. ${ }^{18}$ In a single institution experience reporting outcomes for patients treated with IORT using the Intrabeam (TARGIT) device, all 38 evaluated with ultrasound 6-12 months post-IORT were found to have developed a seroma, and $26 \%$ were symptomatic. ${ }^{19}$

While the increased risk of breast cancer recurrence with IORT is a cause for concern, invariably patient preference will also play a significant role in a patient's treatment decisions. Alvarado et al reported from questionnaires given to 81 patients and found that the majority of breast cancer patients will accept a small increment of local risk for a simpler delivery of radiation. ${ }^{20}$ The predefined noninferiority margin of $2.5 \%$ selected by the TARGIT-A trial would be acceptable by the majority of this group of selected patients, who endorsed a median accepted risk of $2.3 \%$. Not unexpectedly, $91 \%$ of patients would choose IORT over WBI if the recurrence risks were equal, warranting further refinements to IORT and increasing availability of APBI.

\section{Cost}

Grobmyer et al, in a report on their own experience with the IORT using the Intrabeam device, found markedly lower treatment costs with IORT (\$1857) compared to WBI 
(\$9653). ${ }^{21}$ In a similar analysis, Alvarado et al, using a Markov decision-analytic model, reported less cost and greater quality-adjusted life years (QALY) with IORT compared to WBI; however, they noted that due to the higher risk of recurrence IORT should only be applied to the eligible population..$^{22}$ In contrast, in a cost-per-QALY analysis, Shah et al showed that when factoring in additional medical costs, nonmedical costs, and cost of recurrences, three-dimensional conformal radiation therapy and APBI had a lower overall cost compared to IORT performed in the TARGIT-A and ELIOT trials. ${ }^{23}$ While the existing literature is conflicting and reimbursement models are ever changing, it is in general agreed that IORT in properly selected women would offer a net cost savings to the health care system.

\section{Current controversy}

Although prospective randomized Phase III trials are considered the pinnacle of clinical oncology trials, the conclusion from the TARGIT trialists' group that the TARGIT IORT method was noninferior to WBI in the prepathology group has been subject to several criticisms. Wazer and Hepel argued that the follow-up time (median 2.4 years) was too short to evaluate local failure since most failures occur after 5 years for small estrogen receptor-positive tumors and that accurate assessment of late toxicities from high-dose focal irradiation requires at least 5 years of follow-up. ${ }^{24}$ In response, TARGITA trial researchers claimed that most of the reduction in recurrence by radiation therapy is in the first 5 years. ${ }^{25}$

A second argument against TARGIT-IORT was that $15 \%$ of all patients in the TARGIT arm received WBI (nearly all were in the prepathology group); however, Vaidya et al countered that several patients with poor prognosis cancers (grades 2-3, $>1 \mathrm{~cm}$, and nodal involvement) still had excellent local control with TARGIT alone. The TARGIT-A trial protocol provided recommendations for WBI after IORT; however, sites were allowed to customize eligibility criteria. When comparing consensus guidelines for selection criteria between ASTRO, GEC-ESTRO, and the TARGIT group, a single institution found that the rate of WBI recommendation after IORT can vary from $5 \%$ to $33 \%$; however, it would remain $<10 \%$ if no more than one cautionary factor (per ASTRO APBI guidelines) is permitted. ${ }^{26}$

Regarding toxicity, the authors of TARGIT the trial attributed increase in non-breast cancer-related deaths to the toxicities caused by WBI. Yarnold et al argued that causation is unlikely, due to the low expected mean heart dose with current WBI techniques and previous breast cancer trials showing that secondary cancers take $>10$ years to emerge. ${ }^{27} \mathrm{~A}$ meta-analysis of randomized trials comparing APBI to WBI showed a small ( $1.1-1.3 \%)$ but significant $(p=0.23$ ) reduction in non-breast cancer mortality, suggesting under-recognized risks of WBI despite advancement in external beam radiation delivery techniques. ${ }^{28}$ When taking into account the total mortality of $4.9 \%$ among the patients analyzed, a relative reduction of $25 \%$ could be considered clinically significant by many.

A concern of Shah et al was extrapolating typical APBI plans to IORT. ${ }^{29}$ For example, in the TARGIT-A trial, the dose at $1 \mathrm{~cm}$ was markedly lower compared to a typical APBI plan, likely resulting in higher rates of local recurrence. Due to this and above-mentioned flaws regarding the ELIOT trial, ASTRO guidelines, ${ }^{30}$ which group patients into suitability categories for APBI, do not recommend low-energy X-ray IORT outside of prospective registry of clinical trial and state that electron beam IORT should be restricted to patients who meet all "suitable" criteria. Last, the National Surgical Breast and Bowel Project-coordinated prospective trial evaluating APBI (B-39/RTOG 0413) will assist with treatment decisions regarding APBI, although these results are not yet available.

Considering relatively recent widespread adoption of performing sentinel lymph node (SLN) biopsies, results from previous APBI trials are difficult to apply to patients who have positive SLN biopsy but no axillary dissection. For example, Phase II study NRG Oncology/RTOG 9517, ${ }^{31}$ which evaluated interstitial brachytherapy APBI (given over several days), required an axillary node dissection and included patients with three or less positive nodes. Meanwhile, the Phase III GEC-ESTRO randomized trial, which compared APBI using interstitial multicatheter brachytherapy given over multiple days to conventional WBI, required a negative SLN biopsy or axillary dissection, allowing for $\mathrm{N} 0$ or micrometastasis to lymph nodes only to be eligible. Last, the ELIOT trial required axillary node dissection for all patients with a positive SLN biopsy, and $\sim 25 \%$ of participants in the trial were node positive. ASTRO guidelines deem patients with pathologically confirmed nodal disease to be unsuitable for APBI. It is clear that there is considerable variability in the recommendation for IORT in node-positive patients and any such use is considered to be experimental as nodal positivity is a factor known to increase the risk of local recurrence. ${ }^{32}$

\section{Future directions}

The future of IORT is unknown. On the one hand, there exist distinguished scientists and physicians who have valid concerns regarding the safety of IORT. On the other hand, there exists prospective Phase III evidence, along with improved patient satisfaction and decreased costs in an era where these criteria have gained increasing weight in medical decision-making. As 
a result, IORT utilization is increasing nationally in the United States, although National Comprehensive Cancer Network guidelines do not currently include IORT as an acceptable option for breast cancer. ${ }^{33,34}$ However, as described earlier, there are limitations in the TARGIT and ELIOT approaches to IORT. PB-IORT represents an IORT approach that may represent an improvement on conventional IORT techniques as it incorporates HDR brachytherapy and $\mathrm{CT}$ imaging.

An alternative method for delivering IORT is a modified balloon-based APBI called Xoft Axxent electronic brachytherapy, which received US Food and Drug Administration clearance in 2006 and delivers low-energy photons to the lumpectomy cavity surface ( $50 \mathrm{kV}$ energy). ${ }^{35}$ The device uses a mobile controller to generate kilovoltage $\mathrm{X}$-rays, which require minimal shielding and would not require the same radiation source regulations imposed on iridium-192. Ultrasound image guidance is used to measure balloon-to-skin distance and evaluate air or fluid in the surrounding breast tissue.

Currently, preoperative breast MRI is not part of the standard work-up when evaluating patients for BCS and was not required in the TARGIT-A or ELIOT trial. In a highly selected patient population of T1N0 tumors that would be eligible for APBI, Tallet et al showed that preoperative MRI in patient staging leads to diagnosis of an ipsilateral second BC in $4 \%$ of cases. ${ }^{36}$ Intraoperative MRI to assess surgical margins is the topic of the current investigation, ${ }^{37}$ but thus far seems feasible and would theoretically decrease the need for re-excision lumpectomy and/or additional radiotherapy after IORT for close/positive margins, further improving patient convenience.

A major limitation of IORT used in the above TARGIT$A$ and ELIOT trials is image guidance, with the resultant inability to document useful information such as dose to the lumpectomy cavity, but also dose to adjacent structures including the skin, chest wall, lung, and heart. The PB-IORT technique described the above attempts to overcome this limitation by obtaining an intraoperative CT scan, which allows for applicator position adjustment before treatment is delivered. Published data found that the benefit of intraoperative CT was not trivial, in that it identifies actionable findings in breast IORT, including residual tumor or errors in applicator positioning, in $24 \%$ of patients. ${ }^{38}$ These images are also used to aid in optimizing radiation planning and performing dosimetry calculations to organs at risk.

The future of breast IORT may extend beyond BCS. In settings where mastectomy is the preferred surgical approach, such as the case for multifocal tumors, nipple-sparing techniques are sometimes implemented to improve cosmetic outcome. Due to concern for recurrence in the nipple-areolar complex, Petit et al performed a study utilizing the ELIOT device to deliver 16 Gy of IORT to 800 patients undergoing nipple-sparing mastectomy. ${ }^{39}$ There were 13 locoregional recurrences (1.6\%), and none occurred in the nipple-areolar complex. However, complication rate was increased with complete or partial necrosis of the nipple-areolar complex occurring in almost $10 \%$ of patients. As described, the role of IORT int he setting of mastectomy remains to be defined, although has the potential to find a niche that will improve quality of care in this patient population as well.

To date, no subset of patients has been identified that can safely omit adjuvant radiation therapy without the increased risk of local recurrence. Hughes et al demonstrated in a randomized trial that for patients aged $\geq 70$ years with small, estrogen receptor-positive tumors, the addition of radiation therapy to tamoxifen did not improve the overall survival, distant disease-free survival, or breast preservation. ${ }^{40}$ However, a small improvement in locoregional recurrence, 98\% with tamoxifen plus WBI compared to $90 \%$ with tamoxifen alone, was still observed in this low-risk subset of patients. Perhaps, in the future, IORT will find its home in this patient population where the risks and benefits of adjuvant radiotherapy need to be constantly balanced.

There are several groups that are expanding on the currently held notions of IORT, including radiation delivery shortly after surgery. In an effort to evaluate a technique that provides the improved planning and dosimetry available with APBI that further approaches IORT convenience, Stish et al showed feasibility in starting radiation treatments as soon as 1 day following one procedure including lumpectomy, SLN biopsy, and placement of a multichannel brachytherapy catheter. ${ }^{41}$ Other centers typically place the brachytherapy catheter 3-6 weeks following surgery. In this trial, radiotherapy simulation and planning took place the weekday after surgery, and treatment delivery twice a day for 10 days began within 2 weekdays from surgery. Before brachytherapy was initiated, the final pathology was available, and only given if no pathology characteristics deemed them ineligible per institutional protocol.

\section{Conclusion}

IORT appeals to patients because of its significant improvement in convenience. Opportunities to increase dose, improve accuracy, and optimize treatment for tailored treatment planning using treatment technologies that exist within RT armamentarium may improve outcomes after IORT. Further refinement of patient selection for APBI in general and IORT in particular will ultimately define its role. 


\section{Disclosure}

The authors report no conflicts of interest in this work.

\section{References}

1. Consensus statement: treatment of early-stage breast cancer. National Institutes of Health Consensus Development Panel. J Natl Cancer Inst Monogr. 1992;(11):1-5.

2. Fisher B, Anderson S, Bryant J, et al. Twenty-year follow-up of a randomized trial comparing total mastectomy, lumpectomy, and lumpectomy plus irradiation for the treatment of invasive breast cancer. $N \mathrm{Engl}$ J Med. 2002;347(16):1233-1241.

3. Hershman DL, Buono D, McBride RB, et al. Surgeon characteristics and receipt of adjuvant radiotherapy in women with breast cancer. $J$ Natl Cancer Inst. 2008;100(3):199-206.

4. Voti L, Richardson LC, Reis I, Fleming LE, Mackinnon J, Coebergh JW. The effect of race/ethnicity and insurance in the administration of standard therapy for local breast cancer in Florida. Breast Cancer Res Treat. 2006;95(1):89-95.

5. Athas WF, Adams-Cameron M, Hunt WC, Amir-Fazli A, Key CR. Travel distance to radiation therapy and receipt of radiotherapy following breast-conserving surgery. J Natl Cancer Inst. 2000;92(3):269-271.

6. Smith BD, Arthur DW, Buchholz TA, et al. Accelerated partial breast irradiation consensus statement from the American Society for Radiation Oncology (ASTRO). Int J Radiat Oncol Biol Phys. 2009;74(4):987-1001.

7. Shah C, Vicini F, Wazer DE, Arthur D, Patel RR. The American Brachytherapy Society consensus statement for accelerated partial breast irradiation. Brachytherapy. 2013;12(4):267-277.

8. Polgar C, Van Limbergen E, Potter R, et al. Patient selection for accelerated partial-breast irradiation (APBI) after breast-conserving surgery. Recommendations of the Groupe Europeen de Curietherapie-European Society for Therapeutic Radiology and Oncology (GEC-ESTRO) breast cancer working group based on clinical evidence (2009). Radiother Oncol. 2010;94(3):264-273.

9. Veronesi U, Orecchia R, Maisonneuve P, et al. Intraoperative radiotherapy versus external radiotherapy for early breast cancer (ELIOT): a randomised controlled equivalence trial. Lancet Oncol. 2013;14(13):1269-1277.

10. Vaidya JS, Wenz F, Bulsara M, et al. Risk-adapted targeted intraoperative radiotherapy versus whole-breast radiotherapy for breast cancer: 5 -year results for local control and overall survival from the TARGIT-A randomised trial. Lancet. 2014;383(9917):603-613.

11. Sperk E, Welzel G, Keller A, et al. Late radiation toxicity after intraoperative radiotherapy (IORT) for breast cancer: results from the randomized phase III trial TARGIT A. Breast Cancer Res Treat. 2012;135(1):253-260.

12. Showalter SL, Petroni G, Trifiletti DM, et al. A novel form of breast intraoperative radiation therapy with CT-guided high-dose-rate brachytherapy: results of a prospective phase 1 clinical trial. Int J Radiat Oncol Biol Phys. 2016;96(1):46-54.

13. Jones R, Libby B, Showalter SL, et al. Dosimetric comparison of (192)Ir high-dose-rate brachytherapy vs. $50 \mathrm{kV}$ x-rays as techniques for breast intraoperative radiation therapy: conceptual development of imageguided intraoperative brachytherapy using a multilumen balloon applicator and in-room CT imaging. Brachytherapy. 2014;13(5):502-507.

14. Badakhshi H, Gruen A, Sehouli J, Budach V, Boehmer D. The impact of patient compliance with adjuvant radiotherapy: a comprehensive cohort study. Cancer Med. 2013;2(5):712-717.

15. Welzel G, Boch A, Sperk E, et al. Radiation-related quality of life parameters after targeted intraoperative radiotherapy versus whole breast radiotherapy in patients with breast cancer: results from the randomized phase III trial TARGIT-A. Radiat Oncol. 2013;8:9.

16. Corica T, Nowak AK, Saunders CM, et al. Cosmesis and breast-related quality of life outcomes after intraoperative radiation therapy for early breast cancer: a substudy of the TARGIT-A trial. Int J Radiat Oncol Biol Phys. 2016;96(1):55-64.
17. Veronesi $\mathrm{U}$, Orecchia $\mathrm{R}$, Luini A, et al. Intraoperative radiotherapy during breast conserving surgery: a study on 1,822 cases treated with electrons. Breast Cancer Res Treat. 2010;124(1):141-151.

18. Elsberger B, Romsauerova A, Vinnicombe S, et al. Comparison of mammographic findings after intraoperative radiotherapy or external beam whole breast radiotherapy. Eur J Surg Oncol. 2014;40(2):163-167.

19. Goble RN, Drukteinis JS, Lee MC, Khakpour N, Kiluk JV, Laronga C. Early experience with ultrasound features after intrabeam intraoperative radiation for early stage breast cancer. J Surg Oncol. 2014; 109(8):751-755.

20. Alvarado MD, Conolly J, Park C, et al. Patient preferences regarding intraoperative versus external beam radiotherapy following breastconserving surgery. Breast Cancer Res Treat. 2014;143(1):135-140.

21. Grobmyer SR, Lightsey JL, Bryant CM, et al. Low-kilovoltage, singledose intraoperative radiation therapy for breast cancer: results and impact on a multidisciplinary breast cancer program. J Am Coll Surg. 2013;216(4):617-623.

22. Alvarado MD, Mohan AJ, Esserman LJ, et al. Cost-effectiveness analysis of intraoperative radiation therapy for early-stage breast cancer. Ann Surg Oncol. 2013;20(9):2873-2880.

23. Shah C, Badiyan S, Khwaja S, et al. Evaluating radiotherapy options in breast cancer: does intraoperative radiotherapy represent the most cost-efficacious option? Clin Breast Cancer. 2014;14(2):141-146.

24. Hepel J, Wazer DE. A flawed study should not define a new standard of care. Int J Radiat Oncol Biol Phys. 2015;91(2):255-257.

25. Vaidya JS, Bulsara M, Wenz F, et al. Pride, prejudice, or science: attitudes towards the results of the TARGIT-A trial of targeted intraoperative radiation therapy for breast cancer. Int J Radiat Oncol Biol Phys. 2015;92(3):491-497.

26. Mellon EA, Orman A, Joya LE, et al. Frequency of whole breast radiation therapy after intraoperative radiation therapy due to criteria identified by lumpectomy. Brachytherapy. 2016;16(1):174-180.

27. Yarnold J, Offersen BV, Olivotto I, Poortmans P, Sarin R. Radiotherapy for breast cancer, the TARGIT-A trial. Lancet. 2014;383(9930):1717-1718.

28. Vaidya JS, Bulsara M, Wenz F, et al. Reduced mortality with partialbreast irradiation for early breast cancer: a meta-analysis of randomized trials. Int J Radiat Oncol Biol Phys. 2016;96(2):259-265.

29. Shah C, Khan AJ, Arthur D, Fernandez E, Martinez AA, Vicini F. Intraoperative radiation therapy in breast cancer: not ready for prime time. Ann Surg Oncol. 2014;21(2):351-353.

30. Correa C, Harris EE, Leonardi MC, et al. Accelerated partial breast irradiation: executive summary for the update of an ASTRO evidencebased consensus statement. Pract Radiat Oncol. 2016;7(2):73-79.

31. White J, Winter K, Kuske RR, et al. Long-term cancer outcomes from study NRG oncology/RTOG 9517: a phase 2 study of accelerated partial breast irradiation with multicatheter brachytherapy after lumpectomy for early-stage breast cancer. Int J Radiat Oncol Biol Phys. 2016;95(5):1460-1465.

32. Clarke M, Collins R, Darby S, et al. Effects of radiotherapy and of differences in the extent of surgery for early breast cancer on local recurrence and 15-year survival: an overview of the randomised trials. Lancet. 2005;366(9503):2087-2106.

33. Trifiletti DM, Showalter TN, Romano KD, et al. Fractionation trends in breast cancer and implications in partial breast irradiation. J Radiat Oncol. In press 2017.

34. National Comprehensive Cancer Network. Breast Cancer (Version 2.2016). Available from: https://www.nccn.org/professionals/physician_gls/pdf/breast.pdf. Accessed December 26, 2016.

35. Dickler A, Ivanov O, Francescatti D. Intraoperative radiation therapy in the treatment of early-stage breast cancer utilizing Xoft Axxent electronic brachytherapy. World J Surg Oncol. 2009;7:24.

36. Tallet A, Rua S, Jalaguier A, et al. Impact of preoperative magnetic resonance imaging in breast cancer patients candidates for an intraoperative partial breast irradiation. Transl Cancer Res. 2015;4:148-154.

37. Golshan M, Sagara Y, Wexelman B, et al. Pilot study to evaluate feasibility of image-guided breast-conserving therapy in the advanced multimodal image-guided operating (AMIGO) suite. Ann Surg Oncol. 2014; 21(10):3356-3357. 
38. Trifiletti DM, Showalter TN, Libby B, et al. Intraoperative breast radiation therapy with image guidance: findings from CT images obtained in a prospective trial of intraoperative high-dose-rate brachytherapy with CT on rails. Brachytherapy. 2015;14(6):919-924.

39. Petit JY, Veronesi U, Orecchia R, et al. Nipple sparing mastectomy with nipple areola intraoperative radiotherapy: one thousand and one cases of a five years experience at the European institute of oncology of Milan (EIO). Breast Cancer Res Treat. 2009;117(2):333-338.
40. Hughes KS, Schnaper LA, Bellon JR, et al. Lumpectomy plus tamoxifen with or without irradiation in women age 70 years or older with early breast cancer: long-term follow-up of CALGB 9343. J Clin Oncol. 2013; 31(19):2382-2387.

41. Stish BJ, Pafundi DH, Hieken TJ, et al. Feasibility and full-course dosimetry of an intraoperatively placed multichannel brachytherapy catheter for accelerated partial breast irradiation. Brachytherapy. 2016;15(6) 796-803.
Breast Cancer - Targets and Therapy

\section{Publish your work in this journal}

Breast Cancer - Targets and Therapy is an international, peerreviewed open access journal focusing on breast cancer research, identification of therapeutic targets and the optimal use of preventative and integrated treatment interventions to achieve improved outcomes, enhanced survival and quality of life for the cancer patient.

\section{Dovepress}

The manuscript management system is completely online and includes a very quick and fair peer-review system, which is all easy to use. Visit http://www.dovepress.com/testimonials.php to read real quotes from published authors.

Submit your manuscript here: https:/www.dovepress.com/breast-cancer---targets-and-therapy-journal 